\title{
A reversal of the PRE following complex stimulus training
}

\author{
Sally E. Sperling 2 \\ UNIVERSITY OF CALIFORNIA, RIVERSIDE
}

\begin{abstract}
Greater resistance to extinction was observed if the extinction stimulus had been the positive discriminandum during discrimination training with two $50 \%$ reinforced irrelevant stimuli also present than if it had been a 50\% reinforced irrelevant stimulus during discrimination training on another pair of stimuli. Compared to the more usual training conditions, the data seem more likely to reflect an increase in resistance to extinction for the former group than a decrease for the latter.
\end{abstract}

\section{Problem}

At the same time that discrimative control over responding is being acquired by the relevant positive and negative stimuli in a discrimination learning task, some control over responding is also being acquired by stimuli that are associated with reinforcement some \% of the time greater than 0 and less than 100 (Babb, 1956; Jeeves \& North, 1956). Particularly with respect to irrelevant stimuli previously associated with a $50 \%$ reinforcement schedule, the results of an experiment by Sperling, 1962, demonstrated that extinction performance is no different from that obtained following regular $50 \%$ partial reinforcement and suggested that subsequent performance with respect to a previously irrelevant stimulus could be predicted from a knowledge of the past reinforcement history associated with that stimulus.

If this hypothesis is to be entertained for irrelevant stimuli, then it should also be tenable for relevant stimuli, i.e., performance with respect to any stimulus, regardless of its relevant or irrelevant status, should be predictable from past reinforcement history. However, data from the same study showed that resistance to extinction in the presence of a previously positive discriminandum is apparently increased by having $\mathrm{E}$ manipulated $50 \%$ reinforced irrelevant stimuli present during initial training over that exhibited if irrelevant stimuli are not present.

One way of determining whether the concurrent administration of different schedules of reinforcement has different effects on the control over responding acquired by relevant and irrelevant stimuli from those predicted from past reinforcement history is to see whether the phenomena observed following regular continuous or partial reinforcement training are replicable following complex stimulus training, e.g., the partial reinforcement effect (PRE). These data are reported below.

\section{Method}

The Ss were 60 naive male hooded rats, 90 days of age at the start of pretraining. Prior to extinction 3 Ss were discarded because of illness.

The apparatus was two elevated platforms enclosed in a cubicle compartment divided by a partition into starting and stimulus sections. When S broke an infrared light beam at the terminal end of the starting platform, a 100-w bulb over the stimulus compartment was turned on and a timer was started which stopped when $\mathrm{S}$ stepped off the stimulus platform.

Two interchangeable stimulus platforms longitudinally striped with black and white Mystic Tape were used; one with 1-in stripes (W) and one with $1 / 2$-in stripes (N). A $20-\mathrm{v}$ light bulb centered on a swinging door at the terminal end of the stimulus platform could be either on (L) or off (D).

A $23 \mathrm{hr}$.deprivation schedule was maintained throughout the experiment. Following 11 days of pretraining Ss were assigned to one of two experimental groups at one of three levels of training.

L was the relevant positive discriminandum for one group and $\mathrm{D}$ the negative. The $\mathrm{W}$ platform was present on half of the $\mathrm{L}$ and half of the $\mathrm{D}$ trials; the $\mathrm{N}$ platform was present on the other trials. The $29 \mathrm{Ss}$ in this group were divided into three subgroups receiving either 48 trials $(\mathrm{N}=9), 72$ trials $(\mathrm{N}=9)$ or trials to a criterion of not more than one positive trial within a day with a latency longer than any of the negative trials $(N=11)$. These groups are designated 48LDwn, 72LDwn, and CLDwn, respectively, with capital letters indicating the relevant and lower case letters the irrelevant stimuli.

For the other group W was the relevant positive and $\mathrm{N}$ the relevant negative discriminandum, with $\mathrm{L}$ and $\mathrm{D}$ as irrelevant stimuli. Groups 48WNld, 72WNld, and CWNld contained 9, 9, and 10 Ss respectively.

There were 6 positive and 6 negative acquisition trials a day on a 10-min. intertrial interval. Each irrelevant stimulus appeared 3 times each with the positive and with the negative. A food cup was not present on negative trials; on positive trials it was baited with $294 \mathrm{mg}$ Noyes pellets.

Each $\mathrm{S}$ received 15 extinction trials on a 10-min. intertrial interval on the day following acquisition. The swinging door was removed from the apparatus, the $\mathrm{W}$ platform was in place, and the food cup was absent. Results

All data were transformed to $\log$ (latency $x 100$ ) for analysis.

There was no difference between days to criterion for the two $\mathrm{C}$ groups (8.8 and 9.3 days); both of the $\mathrm{C}$ groups were responding significantly faster on positive than on 
negative trials during their last day of acquisition; group 72LDwn also showed this difference.

Since the groups received extinction trials after varying amounts of acquisition training, their respective levels of performance in the presence of $\mathrm{W}$, the stimulus presented during extinction, were compared by an analysis of variance of mean log latency for the 6 trials on which $\mathrm{W}$ appeared as a part of the stimulus complex during the last day of acquisition; $\mathrm{F}(5,51)=3.26 ; \mathrm{p}<.025$. Tukey $95 \%$ confidence intervals showed that group $72 \mathrm{LDwn}$ was responding significantly faster than group CLDwn on these trials; no other differences were significant.

Because not all of the groups could be considered to have entered extinction with common speeds in the presence of $\mathrm{W}$ a separate analysis of variance was computed for the WNld and LDwngroups at each of the three levels of training for extinction trials 2-15. (Since the first trial constituted a stimulus change for all Ss, and since the latency on this trial was recorded prior to the first experience of nonreinforcement in this new situation, the first trial was omitted from the analysis.)

The Fs for the comparison between the 48WNld and $48 \mathrm{LDwn}$ groups and the $72 \mathrm{WNld}$ and $72 \mathrm{LDwn}$ groups were both less than 1 . For the two $C$ groups $F(1,19)=5.62$; $\mathrm{p}<.05$, with group CWNld responding significantly faster than group CLDwn.

\section{Diseussion}

The 48-trial groups served as a check on performance early in training, and no differences were found at that level. The data for the 72-trial groups, both in absolute level of responding during acquisition and extinction and the lack of the PRE, were comparable to those obtained for two 80-trial groups in Sperling, 1962.

The greater resistance to extinction found in CWNld over CLDwn could be explained on the basis that irrelevant stimuli cease to function as effective parts of a stimulus complex with increased amounts of training and discriminative responding in the presence of the relevant stimuli (e.g., Restle, 1957; Lawrence, 1949,1950). However, within-group rank order correlation coefficients of .81 in each group indicate that the greater the discriminative responding on the last day of acquisition, as indexed by the difference between positive and negative latencies, the faster the responding during extinction, regardless of the previously relevant or irrelevant status of the extinction stimulus. Therefore, the data suggest that the discrepancy between these findings and the usual PRE results is due to increased resistance to extinction in CWNld rather than decreased resistance to extinction in CLDwn.

The explanation for the increased resistance to extinction in the former group must lie in the effect of pairing the presentation of the positive discriminandum with that of a $50 \%$ reinforced irrelevant stimulus on reinforced trials. Since the increased resistance to extinction is not observable until after a reasonable amount of training, some mechanism like Amsel's $r_{f}$ (1962) is suggested.

\section{References}

AMSEL, A. Frustrative nonreward in partial reinforcement and discrimination learning: Some recent history and a theoretical extension. Psychol. Rev., 1962, 69, 306-328.

BABB, H. Proportional reinforcement of irrelevant stimuli. J. comp. physiol. Psychol., 1956, 49, 586-589.

JEEVES, M. A., \& NORTH, A. J. Irrelevant or partially correlated stimuli in discrimination learning. J. exp. Psychol., 1956, 52, 90-94.

LAWRENCE, D. H. Acquired distinctiveness of cues: I. Transfer between discriminations on the basis of familiarity with the stimulus. J. exp. Psychol., 1949, 39, 770-785.

LAWRENCE, D. H. Acquired distinctiveness of cues: II. Selective association in a constant stimulus situation. J. exp. Psychol., 1950, 40, 175-189.

RESTLE, F. Discrimination of cues in mazes: A resolution of the "place-vs-response" controversy. Psychol. Rev., 1957, 65, 217-228.

SPERLING, S. E. Extinction effects following nondifferential reinforcement of an irrelevant stimulus. J. exp. Psychol., 1962, 63, 50-56.

\section{Notes}

1. This research was conducted under grant G-24168 from the National Science Foundation.

2. The author is indebted to Fred Valle, Michael Miller, and Peter Brill for their assistance in data collection and tabulation. 